Case Study

\title{
Common Diatoms of Phytobenthos in Gediz River Basin
}

\section{Gediz Nehir Havzasındaki Fitobentozun Yaygın Diyatomeleri}

\author{
Cüneyt Nadir SOLAK ${ }^{1}$, Tolga ÇETIN ${ }^{2}$, Yakup KARAASLAN ${ }^{2}$, Aydın KALELI $^{1}$, Elif YILMAZ ${ }^{1}$, Mustafa \\ DURAN $^{3}$, Gürçay KIVANÇ ${ }^{3}$, Taner KIMENÇE ${ }^{2}$, Şebnem AYNUR KOYUNLUOĞLU ${ }^{4}$, Burhan Fuat \\ ÇANKAYA ${ }^{2}$, Deniz YILMAZ AŞIK ${ }^{2}$ \\ ${ }^{1}$ Dumlupinar University, Faculty of Arts and Sciences, Department of Biology, Kütahya, Republic of Turkey \\ cnsolak@gmail.com, aydinkaleli84@gmail.com, elfyilmaz38@gmail.com \\ ${ }^{2}$ Ministry of Agriculture and Forestry, General Directorate of Water Management, Ankara, Republic of \\ Turkey \\ tolga.cetin@tarimorman.gov.tr, yakup.karaaslan@tarimorman.gov.tr, taner.kimence@tarimorman.gov.tr \\ burhanfuat.cankaya@tarimorman.gov.tr,yilmazasik.deniz@tarimorman.gov.tr \\ ${ }^{3}$ Pamukkale University, Faculty of Arts and Sciences, Hydrobiology Laboratory, Kinikli Campus, 20070 \\ Denizli, Republic of Turkey \\ mduran@pau.edu.tr, gkakyildiz@pau.edu.tr \\ ${ }^{4}$ TÜBITTAK MAM, Environment and Cleaner Production Institute, Gebze/KOCAELI, Republic of Turkey \\ sebnem.aynur@tubitak.gov.tr
}

Received Date: 4.12.2018, Accepted Date: 24.12.2018

\begin{abstract}
Diatoms (important representatives of phytobenthos) are ecologically significant quality elements for rivers and lakes according to the EU Water Framework Directive (2000/60/EC). We aimed to investigate common diatoms of Gediz River Basin within the scope of the studies for preparation of Gediz River Basin Management Plan. Samples were collected from epilithon and epipelon substrates of 17 rivers, 2 lakes (Gölcük and Marmara) and 4 dams (Demirköprü, Küçükler, Buldan, Afşar) from November 2017 to April 2018. The samples were boiled with $\mathrm{H}_{2} \mathrm{O}_{2}$ and $\mathrm{HCl}$ for removing the organic matter from frustules. Permanent slides were mounted with Naphrax solution. As a result, 28 taxa were found as common diatoms of Gediz River Basin. Many of the taxa were commonly found also in Turkish rivers and lakes. Amphora pediculus (Kützing) Grunow, Navicula reichardtiana Lange-Bertalot, Navicula veneta Kützing, Nitzschia dissipata (Kützing) Rabenhorst and Nitzschia inconspicua Grunow were most common diatoms in sampling points. On the other hand, Navicula erifuga Lange-Bertalot, Navicula reichardtiana Lange-Bertalot and Nitzschia archibaldii Lange-Bertalot were rarely found in Turkish rivers and lakes.
\end{abstract}

Keywords: Common diatoms, Gediz River Basin, phytobenthos

$\ddot{O} \mathbf{z}$

AB Su Çerçeve Direktifine (2000/60/EC) göre; fitobentozun önemli temsilcilerinden olan diatomlar, nehir ve göller için önemli ekolojik kalite göstergelerindendir Gediz Havzası Yönetim Planı hazırlanması çalışmaları kapsamında Gediz Nehir Havzası için yaygın diyatomelerin araştırılmasını amaçladık. Örnekler 17 nehir, 2 göl (Gölcük ve Marmara) ve 4 barajdan (Demirköprü, Küçükler, Buldan, Afşar) Kasım 2017 ve Nisan 2018 'de epiliton ve epipelon substratlardan toplanmıştır. Organik maddenin frustullerden uzaklaştırılması için örnekler $\mathrm{H}_{2} \mathrm{O}_{2}$ ve $\mathrm{HCl}$ ile kaynatılmıştır. Daimi preparatlar Naphrax solüsyonu ile hazırlanmıştır. Sonuç olarak, Gediz Nehir Havzasında 28 diyatome taksonu yaygın olarak bulunmuştur. Taksonların pek çoğu Türkiye nehir ve göllerinde yaygın olarak bulunan taksonlardır. Amphora pediculus (Kützing) Grunow, Nitzschia dissipata (Kützing) Rabenhorst ve Nitzschia inconspicua Grunow örnekleme noktalarında tespit edilen en yaygın türler olmuştur. Ancak, Navicula erifuga Lange-Bertalot, Navicula reichardtiana Lange-Bertalot ve Nitzschia archibaldii Lange-Bertalot Türkiye nehir ve gölleri için nadir bulunan türlerdendir.

Anahtar kelimeler: Yaygın diyatomeler, Gediz Nehir Havzası, fitobentoz 


\section{Introduction}

Water Framework Directive (2000/60/EC) (Anonymous, 2000) aims to establish a framework for the protection of rivers, lakes, transitional waters, coastal waters and groundwater. Member States shall ensure that a river basin management plan is produced for each river basin. Establishment of river basin management plans has been accelerated last years in Turkey. Turkey has 25 river basins and for 11 river basins (Konya, Susurluk, Meriç-Ergene, Büyük Menderes, Gediz, Yeşilırmak, Akarçay, Batı Akdeniz, Burdur, Küçük Menderes, Kuzey Ege) preparation of river basin management plans are still ongoing.

A river basin management plan shall cover the surface waters ecological status. Ecological status is an expression of the quality of the structure and functioning of aquatic ecosystems associated with surface waters. Phytoplankton, macrophyte and phytobenthos, benthic invertebrates and fish are the biological quality elements for the classification of ecological status (Anonymous, 2000). There are some studies (Demir et al., 2017; Çelekli et al., 2018; Solak et al., 2018a) for identifying the ecological status of water bodies in Turkey within the scope of the river basin based monitoring studies.

Regarding the diatom studies of Gediz River Basin, some works were done in the basin. Karagöl was one of the pioneer works for Turkish inland waters by Güner (1969). Then, Marmara Lake was investigated by Cirik $(1983,1994)$ and Demirköprü Reservoir was worked by GezerlerŞipal et al. (1999) in the basin. Also, Gürle River was examined by Yurterin \& Öztürk (2001) as lotic system. This study aims to investigate the distribution of common benthic diatoms in the Gediz River Basin.

\section{Method}

\section{Study Site}

Gediz River Basin is located between $38^{\circ} 04^{\prime}-39^{\circ}-13^{\prime}$ northern latitudes with $26^{\circ}$ $42^{\prime}-29^{\circ} 45^{\prime}$ eastern longitudes. The main water source of the basin is Gediz River. The Gediz River is $275 \mathrm{~km}$. long and is fed by $17220 \mathrm{~km}^{2}$ of precipitation area in Western Anatolia. There are very few natural lakes in the basin. The most important natural lake is Marmara. The minimum and maximum elevation of the lake is $73.2-79.2 \mathrm{~m}$ and the minimum and maximum volume is $8.5-320 \mathrm{hm}^{3}$. There is Gölcük Lake as a natural lake in the basin except Lake Marmara. This lake is a crater lake, 80 ha wide and $10 \mathrm{~m}$ deep.

There are 5 dams in the river basin. The larger dam in the basin is Demirköprü Dam with a storage capacity of 1.022 million $\mathrm{m}^{3}$. A hydroelectric power plant was established to produce energy on the dam. Küçükler Dams are also used for drinking water supply. The other dams are used for irrigation, flood protection and energy production. Demirköprü, Afşar and Buldan Dams are located in Manisa and Küçükler Dam is located in Uşak (Tarım ve Orman Bakanlığı, Su Yönetimi Genel Müdürlüğü, 2018). 


\section{Sampling}

Samples were collected from 17 rivers, 2 lakes (Gölcük and Marmara) and 4 dams (Demirköprü, Küçükler, Buldan, Afşar) (Table 1) in Gediz River Basin (Figure 1) in November 2017 and April 2018 from 23 sampling points. Epilithic samples were taken by brushing the submerged stones and epipelic samples were taken by using a pipette aspirator from the sediment.

Table 1

The Coordinates of the Sampling Points of the Water Bodies in Gediz River Basin

\begin{tabular}{|c|c|c|c|}
\hline \multirow{2}{*}{ Stations } & \multirow{2}{*}{ Water Bodies } & \multicolumn{2}{|c|}{ Coordinates } \\
\hline & & $\mathbf{x}$ & $\mathbf{Y}$ \\
\hline 1 & G1-Buldan Reservoir & $38^{\circ} 09^{\prime} 25.235^{\prime \prime}$ & $28^{\circ} 50^{\prime} 41.002^{\prime \prime}$ \\
\hline 2 & G2-Demirköprü Reservoir & $38^{\circ} 39^{\prime} 56.785^{\prime \prime}$ & $28^{\circ} 22^{\prime} 14.717^{\prime \prime}$ \\
\hline 3 & G3-Marmara Lake & $38^{\circ} 36^{\prime} 54.525^{\prime \prime}$ & $28^{\circ} 00^{\prime} 47.469^{\prime \prime}$ \\
\hline 4 & G4-Gölcük Lake & $38^{\circ} 19^{\prime} 02.038^{\prime \prime}$ & $28^{\circ} 01^{\prime} 30.273^{\prime \prime}$ \\
\hline 5 & G5-Küçükler Reservoir & $38^{\circ} 52^{\prime} 21.664^{\prime \prime}$ & $29^{\circ} 37^{\prime} 08.719^{\prime \prime}$ \\
\hline 6 & G6-Afşar 2 Reservoir & $38^{\circ} 13^{\prime} 59.251^{\prime \prime}$ & $28^{\circ} 35^{\prime} 50.661^{\prime \prime}$ \\
\hline 7 & N1-Demirci Stream & $38^{\circ} 47^{\prime} 51.690^{\prime \prime}$ & $28^{\circ} 29^{\prime} 54.949^{\prime \prime}$ \\
\hline 8 & N2-Gürdük Stream & $39^{\circ} 03^{\prime} 13.752^{\prime \prime}$ & $27^{\circ} 55^{\prime} 21.694^{\prime \prime}$ \\
\hline 9 & N3-Gediz River & $38^{\circ} 40^{\prime} 55.351^{\prime \prime}$ & $27^{\circ} 21^{\prime} 44.960^{\prime \prime}$ \\
\hline 10 & N4-Gediz River & $38^{\circ} 36^{\prime} 06.540^{\prime \prime}$ & $28^{\circ} 48^{\prime} 46.854^{\prime \prime}$ \\
\hline 11 & N5-Gürlevik Stream & $38^{\circ} 28^{\prime} 58.543^{\prime \prime}$ & $27^{\circ} 49^{\prime} 59.015^{\prime \prime}$ \\
\hline 12 & N6-Bahçeler Stream & $38^{\circ} 58^{\prime} 32.408^{\prime \prime}$ & $29^{\circ} 23^{\prime} 31.106^{\prime \prime}$ \\
\hline 13 & N7-Diken Stream & $38^{\circ} 45^{\prime} 12.438^{\prime \prime}$ & $29^{\circ} 11^{\prime} 36.873^{\prime \prime}$ \\
\hline 14 & N8-Derbent Stream & $38^{\circ} 10^{\prime} 55.692^{\prime \prime}$ & $28^{\circ} 32^{\prime} 35.872^{\prime \prime}$ \\
\hline 15 & N9-Selendi Stream & $38^{\circ} 44^{\prime} 27.451^{\prime \prime}$ & $28^{\circ} 51^{\prime} 59.706^{\prime \prime}$ \\
\hline 16 & N10-Alaşehir Stream & $38^{\circ} 30^{\prime} 27.337^{\prime \prime}$ & $28^{\circ} 08^{\prime} 55.644^{\prime \prime}$ \\
\hline 17 & N11-Gediz River & $38^{\circ} 36^{\prime} 55.182^{\prime \prime}$ & $27^{\circ} 33^{\prime} 15.366^{\prime \prime}$ \\
\hline 18 & N12-Nif Stream & $38^{\circ} 34^{\prime} 15.362^{\prime \prime}$ & $27^{\circ} 34^{\prime} 11.112^{\prime \prime}$ \\
\hline 19 & N13-Sarik1z Springs & $38^{\circ} 46^{\prime} 16.611^{\prime \prime}$ & $27^{\circ} 39^{\prime} 49.604^{\prime \prime}$ \\
\hline 20 & N14-Canburt Stream & $38^{\circ} 39^{\prime} 07.524^{\prime \prime}$ & $27^{\circ} 01^{\prime} 44.680^{\prime \prime}$ \\
\hline 21 & N15-Murat Stream & $38^{\circ} 58^{\prime} 12.143^{\prime \prime}$ & $29^{\circ} 42^{\prime} 53.063^{\prime \prime}$ \\
\hline 22 & N16-Ağ11 Stream & $39^{\circ} 04^{\prime} 27.436^{\prime \prime}$ & $28^{\circ} 40^{\prime} 20.049^{\prime \prime}$ \\
\hline 23 & N17-Gürdük Stream & $39^{\circ} 08^{\prime} 13.548^{\prime \prime}$ & $28^{\circ} 00^{\prime} 24.678^{\prime \prime}$ \\
\hline
\end{tabular}




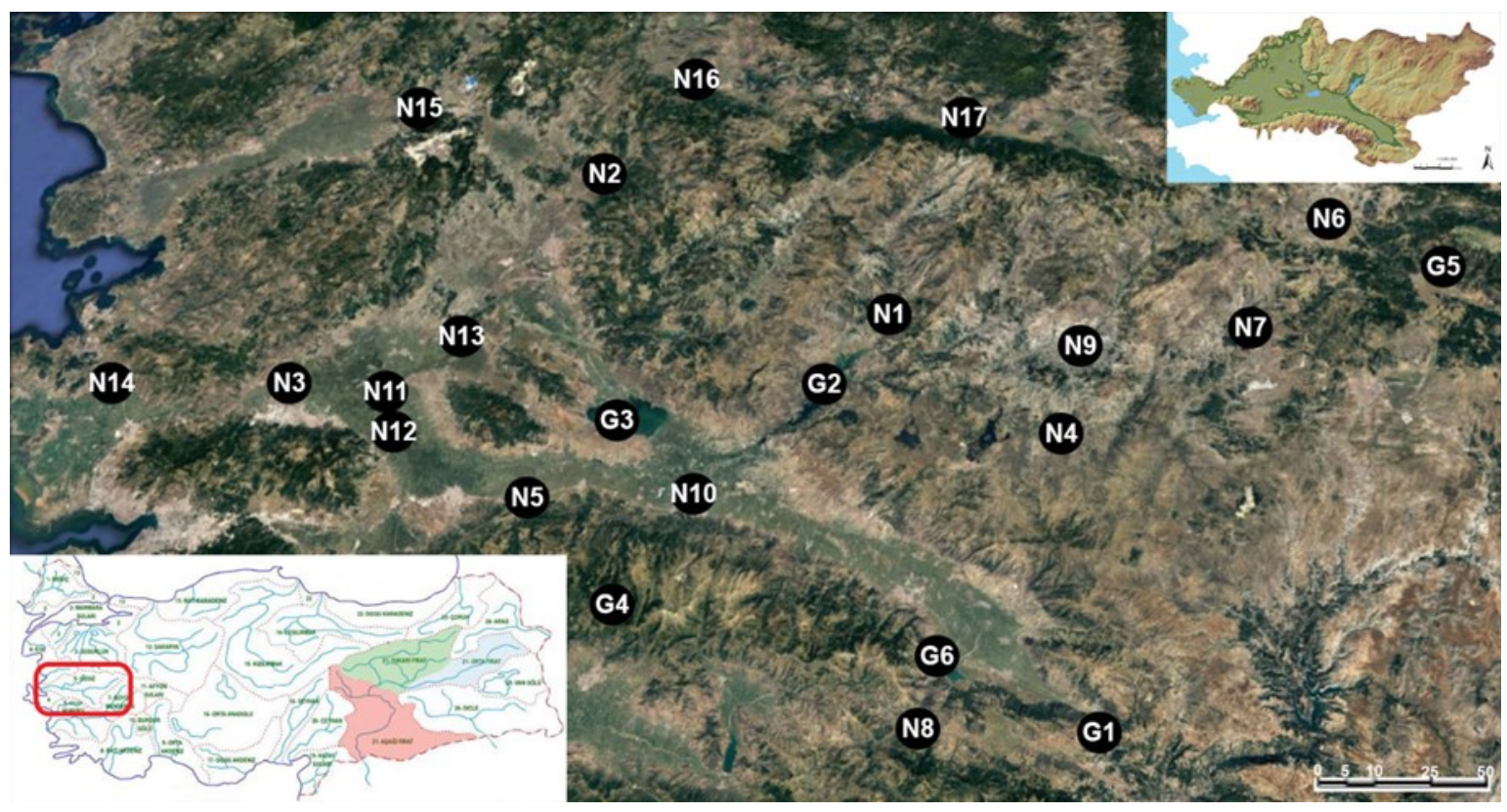

Figure 1. Distribution of sampling points in Gediz River Basin.

\section{Sample Processing, Observation and Identification}

Samples were boiled with $\mathrm{H}_{2} \mathrm{O}_{2}$ and $\mathrm{HCl}$ to remove the organic matter from frustules (Swift 1967). After washing three times of diatoms with distilled water, the material was air-dried on cover glasses and mounted with Naphrax solution. Diatoms were observed with a Nikon Ci Light Microscope (LM) in Dumlupinar University, Turkey. The literature used for identification and dimensions (Krammer 2002, Krammer \& Lange-Bertalot 1986, 1991, Lange-Bertalot et al. 2017).

\section{Diatom Distribution}

Description of the distribution of Turkish diatom flora was made according to Gönülol (2018). The taxa reported from Turkey by Gönülol (2018) which cover $10 \%$ of the literature are categorized as "common" diatoms, the ones cover less than $10 \%$ are noted as "rare" diatoms. If a taxon exists in more than 10 stations, it was named as "common" in this study.

\section{Results}

In this work, common diatoms of Gediz River Basin was evaluated and as a result, totally 28 taxa were commonly found in the sampling stations (Table 2, Figure 2).

\section{Amphora pediculus (Kützing) Grunow}

Ref. Hustedt 1930 (p. 343, Fig. 629); Patrick \& Reimer 1975 (p. 253, Fig. 16: 9 - 10); Krammer \& Lange-Bertalot 1986 (p. 346, Fig. 150: 8 - 13); Levkov 2009 (p. 101, Figs. 55: 31 - 34; 78: 40 - 47); Hofmann et al. 2011 (p. 98, Fig. 91: 29 - 33). 


\section{Cocconeis pediculus Ehrenberg}

Ref. Hustedt 1930 (p. 188, Fig. 259); Patrick \& Reimer 1966 (p. 240, Fig. 15: 3 - 4); Krammer \& Lange-Bertalot 1991 (p. 88, Fig. 57: 1 -4); Hofmann et al. 2011 (p. 132, Fig. 19: $17-19)$.

\section{Cyclotella meneghiniana Kützing}

Ref. Krammer and Lange-Bertalot, 1991 (p. 44, fig. 44: 1 - 10); Håkansson, 2002 (p. 79, figs. 263 - 268); Wojtal and Kwandrans, 2006 (p. 186, fig. 4: 18 - 21, 7: 1 - 13, 9: 1 - 8, 10: 1 - 5); Kiss et al., 2012 (p. 337, fig. 14: A - C); Bey and Ector, 2013 (Vol. 1, p. 30); Cavalcante et al., 2013 (p. 243, fig. 8: A - O); Houk et al., 2010 (p. 16, fig. 143: 1 - 15).

\section{Cymbella excisa Kützing}

Ref. Krammer 2002 (p. 26, pl. 8: 1 - 26); Hofmann et al. 2011 (p. 150, pl. 77: 23 - 28); Bąk et al. 2012 (p. 81, pl. 54).

\section{Diatoma moniliformis (Kützing) D.M.Williams}

Ref. Hofmann et al. 2011 (p. 174, pl. 2: 11 - 15); Bąk et al. 2012 (p. 99, pl. 4).

\section{Encyonema minutum (Hilse) D.G.Mann}

Ref. Krammer 1997 (p.53-pl.25:1-19); Hofmann et al. 2011 (p. 188, pl. 87: 33 - 40); Bąk et al. 2012 (p. 111, pl. 61).

\section{Epithemia sorex Kützing}

Ref. Hustedt 1930 (p. 388, Fig. 736); Patrick \& Reimer 1975 (p. 188, Fig. 27: 4); Krammer \& Lange-Bertalot 1988 (p. 154, Fig. 106: 1 - 13); Hofmann et al. 2011 (p. 206, Fig. 121: 1 $-7)$

\section{Gomphonema olivaceum (Hornemann) Brébisson}

Ref. Hustedt 1930 (p. 378, Fig. 719); Patrick \& Reimer 1975 (p. 139, Fig. 8: 13 - 14); Krammer \& Lange-Bertalot 1986 (p. 374, Fig. 165: 1 - 8); Hofmann et al. 2011 (p. 310 , Fig. 95: $1-6)$.

\section{Gomphonema parvulum (Kützing) Kützing}

Ref. Hustedt 1930 (p. 372, Fig. 713a); Patrick \& Reimer 1975 (p. 122, Fig. 17: 7 - 12); Krammer \& Lange-Bertalot 1986 (p. 358, Fig. 154: 1 - 25); Hofmann et al. 2011 (p. 312, Fig. 99: $1-10)$.

\section{Melosira varians C.Agardh}

Ref. Krammer and Lange-Bertalot, 1991 (p. 7, fig. 4: 1 - 8); Wojtal, 2009 (p. 238, fig. 1: 1 -4); Bey and Ector, 2013 (Vol. 1, p. 48); Cavalcante et al., 2013 (p. 246, fig. 11: A).

\section{Navicula capitatoradiata H.Germain}

Ref. Hofmann et al. 2011 (p. 374, pl. 36: 28 - 34); Bąk et al. 2012 (p. 215, pl. 31).

\section{Navicula cryptotenella Lange-Bertalot}

Ref. Hofmann et al. 2011 (p. 378, pl. 32: 1 - 5); Bąk et al. 2012 (p. 217, pl. 29). 


\section{Navicula tripunctata (O.F.Müller) Bory}

Ref. Hustedt 1930 (p. 299, Fig. 514); Patrick \& Reimer 1966 (p. 513, Fig. 49: 3); Krammer \& Lange-Bertalot 1986 (p. 95, Fig. 27: 1 - 3); Lange-Bertalot 2001 (p. 73, Fig. 1: 1 - 8); Hofmann et al. 2011 (p. 403, Fig. 35: $11-16$ ).

\section{Navicula veneta Kützing}

Ref. Hustedt 1930 (p. 295, Fig. 497a); Patrick \& Reimer 1966 (p. 504, Fig. 48: 5); Krammer \& Lange-Bertalot 1986 (p. 104, Fig. 32: 1 - 4); Lange-Bertalot 2001 (p. 74, Fig. 14: 23 -30); Hofmann et al. 2011 (p. 406, Fig. 9: 8-12).

\section{Nitzschia archibaldii Lange-Bertalot}

Ref. Krammer \& Lange-Bertalot 1991 (p. 115, pl. 81: 10 - 12), Hofmann et al. 2011 (p. 435, pl. 111: $30-34)$.

\section{Nitzschia capitellata Hustedt}

Ref. Krammer \& Lange-Bertalot 1991 (p. 88, pl. 62: 1 - 12), Hofmann et al. 2011 (p. 438, pl. 113: $11-16)$.

\section{Nitzschia dissipata (Kützing) Rabenhorst}

Ref. Hustedt 1930 (p. 412, Fig. 789); Krammer \& Lange-Bertalot 1988 (p. 19, Fig. 11: 17); Hofmann et al. 2011 (p. 441, Fig. 109: 8-18).

\section{Nitzschia fonticola (Grunow) Grunow}

Ref. Hustedt 1930 (p. 415, Fig. 800); Krammer \& Lange-Bertalot 1988 (p. 103, Fig. 75: 122); Hofmann et al. 2011 (p. 259, Fig. 9: 8-12).

\section{Nitzschia inconspicua Grunow}

Ref. Krammer \& Lange-Bertalot 1991 (p. 95 - pl. 69: 1-13); Hofmann et al. 2011 (p. 446 pl. 112: 35-40).

\section{Nitzschia intermedia Hantzsch}

Ref. Hofmann et al. 2011 (p. 449, pl. 107: 1-6); Bąk et al. 2012 (p. 251, pl. 72).

\section{Nitzschia linearis W.Smith}

Ref. Hustedt 1930 (p. 409, Fig. 784); Krammer \& Lange-Bertalot 1988 (p. 69, Fig. 55: 1 4); Hofmann et al. 2011 (p. 452, Fig. 106: 1 -3).

\section{Nitzschia media Hantzsch}

Ref. Krammer \& Lange-Bertalot 1991 (p. 19, pl. 11: 8 - 14), Hofmann et al. 2011 (p. 441: $14-18)$.

\section{Nitzschia palea (Kützing) W.Smith}

Ref. Hustedt 1930 (p. 416, Fig. 801); Krammer \& Lange-Bertalot 1988 (p. 85, Figs 59: 1 24; 60: 1 - 6); Hofmann et al. 2011 (p. 454, Fig. 111: 1 - 20).

\section{Tryblionella apiculata W.Gregory}

Ref. Krammer \& Lange-Bertalot 1991 (p. 43, pl. 35: 1 - 6); Hofmann et al. 2011 (p. 439, pl. 104: 18 - 22); Bąk et al. 2012 (p. 246, pl. 71). 
Table 2

Common and Rare Diatoms in Turkey (Gönülol, 2018) and in This Study

\begin{tabular}{|c|c|c|c|}
\hline & & & In this study \\
\hline & Status in Turkey & Status & Station(s) \\
\hline $\begin{array}{l}\text { Amphora pediculus } \\
\text { (Kützing) Sranoux }\end{array}$ & $\mathrm{C}$ & $\mathrm{C}$ & $\begin{array}{c}\mathrm{G} 2, \mathrm{G} 3, \mathrm{G} 4, \mathrm{G} 5, \mathrm{G} 6, \mathrm{N1}, \mathrm{N} 2, \mathrm{~N} 4, \mathrm{~N} 5, \mathrm{~N} 7, \mathrm{~N} 8, \mathrm{N9}, \\
\mathrm{N} 10, \mathrm{~N} 11, \mathrm{~N} 12, \mathrm{~N} 15, \mathrm{~N} 16, \mathrm{~N} 17\end{array}$ \\
\hline $\begin{array}{l}\text { Cocconsis pediculus. } \\
\text { Ehrenberg }\end{array}$ & $\mathrm{C}$ & $\mathrm{C}$ & $\begin{array}{c}\mathrm{N} 3, \mathrm{~N} 4, \mathrm{~N} 5, \mathrm{~N} 6, \mathrm{~N} 7, \mathrm{~N} 8, \mathrm{~N} 9, \mathrm{~N} 10, \mathrm{~N} 11, \mathrm{~N} 12 \\
\mathrm{~N} 15, \mathrm{~N} 16, \mathrm{~N} 17\end{array}$ \\
\hline $\begin{array}{l}\text { Cuclotella moneghiviana } \\
\text { Kützing }\end{array}$ & $\mathrm{C}$ & $\mathrm{C}$ & G3, G4, G6, N3, N4, N6, N8, N9, N11, N14 \\
\hline $\begin{array}{l}\text { Cumbella excisa } \\
\text { Kützing }\end{array}$ & $\mathrm{C}$ & $\mathrm{C}$ & $\begin{array}{c}\mathrm{Gl}, \mathrm{G} 2, \mathrm{G} 3, \mathrm{~N} 2, \mathrm{~N} 3, \mathrm{~N} 5, \mathrm{~N} 7, \mathrm{~N} 8, \mathrm{~N} 9, \mathrm{~N} 11, \mathrm{~N} 12 \\
\mathrm{~N} 15, \mathrm{~N} 17\end{array}$ \\
\hline $\begin{array}{l}\text { Diatoma monilifoconis } \\
\text { (Kütring) DMLWilliams. }\end{array}$ & $\mathrm{C}$ & $\mathrm{C}$ & $\begin{array}{c}\mathrm{N1}, \mathrm{N} 2, \mathrm{~N} 3, \mathrm{~N} 4, \mathrm{~N} 5, \mathrm{~N} 6, \mathrm{~N} 7, \mathrm{~N} 9, \mathrm{~N} 11, \mathrm{~N} 12, \mathrm{~N} 15, \\
\mathrm{~N} 16, \mathrm{~N} 17\end{array}$ \\
\hline $\begin{array}{l}\text { Encionsma minutum } \\
\text { (Hillse) D G G Mann }\end{array}$ & $\mathrm{C}$ & $\mathrm{C}$ & $\mathrm{Gl}, \mathrm{G} 3, \mathrm{G} 6, \mathrm{~N} 3, \mathrm{~N} 5, \mathrm{~N} 6, \mathrm{~N} 8, \mathrm{~N} 9, \mathrm{~N} 10, \mathrm{~N} 11, \mathrm{~N} 15$ \\
\hline $\begin{array}{l}\text { Evithewia soxex } \\
\text { Kützing }\end{array}$ & $\mathrm{C}$ & $\mathrm{C}$ & $\mathrm{G} 1, \mathrm{G} 2, \mathrm{G} 3, \mathrm{G} 4, \mathrm{G} 5, \mathrm{~N} 5, \mathrm{~N} 12, \mathrm{~N} 15, \mathrm{~N} 16, \mathrm{~N} 17$ \\
\hline $\begin{array}{l}\text { Gonsphonemua alivaceun } \\
\text { (Hornemann) Brebisson }\end{array}$ & $\mathrm{C}$ & $\mathrm{C}$ & $\begin{array}{c}\mathrm{Gl}, \mathrm{N} 1, \mathrm{~N} 2, \mathrm{~N} 3, \mathrm{~N} 4, \mathrm{~N} 5, \mathrm{~N} 6, \mathrm{~N} 7, \mathrm{~N} 8, \mathrm{~N} 9, \mathrm{~N} 11, \\
\mathrm{~N} 12, \mathrm{~N} 15, \mathrm{~N} 16, \mathrm{~N} 17\end{array}$ \\
\hline $\begin{array}{l}\text { Goupsphonexua parawlum } \\
\text { (Kützing) Kützing }\end{array}$ & $\mathrm{C}$ & $\mathrm{C}$ & $\begin{array}{c}\mathrm{G} 6, \mathrm{Nl}, \mathrm{N4}, \mathrm{N6}, \mathrm{N7}, \mathrm{N8}, \mathrm{N9}, \mathrm{N10}, \mathrm{N11}, \mathrm{N} 12 \\
\mathrm{N14}, \mathrm{N} 16\end{array}$ \\
\hline $\begin{array}{l}\text { Molasiza vaxians } \\
\text { Cagardh. }\end{array}$ & $\mathrm{C}$ & $\mathrm{C}$ & $\mathrm{G} 2, \mathrm{G} 5, \mathrm{G} 6, \mathrm{N1}, \mathrm{N} 3, \mathrm{~N} 4, \mathrm{~N} 6, \mathrm{~N} 8, \mathrm{N9}, \mathrm{N1} 1, \mathrm{~N} 14$ \\
\hline $\begin{array}{l}\text { Nasicula canitatoxadiata } \\
\text { H.Gernain }\end{array}$ & $\mathrm{C}$ & $\mathrm{C}$ & $\begin{array}{c}\mathrm{Gl}, \mathrm{G} 2, \mathrm{G} 3, \mathrm{G} 5, \mathrm{G} 6, \mathrm{~N} 2, \mathrm{~N} 4, \mathrm{~N} 5, \mathrm{~N} 7, \mathrm{~N} 8, \mathrm{N9}, \\
\mathrm{N1} 1, \mathrm{~N} 12, \mathrm{N16}, \mathrm{N17}\end{array}$ \\
\hline $\begin{array}{l}\text { Nawicula cwptotenella } \\
\text { Lange-Bertalot }\end{array}$ & $\mathrm{C}$ & $\mathrm{C}$ & $\mathrm{G} 4, \mathrm{G} 5, \mathrm{G} 6, \mathrm{N1}, \mathrm{N} 2, \mathrm{~N} 4, \mathrm{~N} 5, \mathrm{~N} 7, \mathrm{N9}, \mathrm{N} 10$ \\
\hline $\begin{array}{l}\text { Nowicula exifuga } \\
\text { Lange-Bertalot }\end{array}$ & $\mathrm{R}$ & $\mathrm{C}$ & $\mathrm{Gl}, \mathrm{G} 6, \mathrm{N1}, \mathrm{N} 2, \mathrm{~N} 4, \mathrm{~N} 8, \mathrm{~N} 9, \mathrm{~N} 11, \mathrm{~N} 12, \mathrm{~N} 14$ \\
\hline $\begin{array}{l}\text { Aawicula gregaria } \\
\text { Donkin }\end{array}$ & $\mathrm{C}$ & $\mathrm{C}$ & $\begin{array}{c}\mathrm{G} 6, \mathrm{~N} 1, \mathrm{~N} 2, \mathrm{~N} 3, \mathrm{~N} 4, \mathrm{~N} 5, \mathrm{~N} 7, \mathrm{~N} 8, \mathrm{~N} 9, \mathrm{~N} 10, \mathrm{N11} \\
\mathrm{N} 12, \mathrm{N16}\end{array}$ \\
\hline $\begin{array}{l}\text { Aaxicula novassiberica } \\
\text { Lange-Bertalot }\end{array}$ & $\mathrm{C}$ & $\mathrm{C}$ & $\begin{array}{c}\mathrm{Gl}, \mathrm{G} 2, \mathrm{G} 6, \mathrm{N2}, \mathrm{N} 3, \mathrm{~N} 4, \mathrm{~N} 5, \mathrm{~N} 8, \mathrm{~N} 9, \mathrm{N1} 0, \mathrm{N11} \\
\mathrm{N} 12, \mathrm{~N} 15\end{array}$ \\
\hline $\begin{array}{l}\text { Aasicula veichavdtiona } \\
\text { Lange-Bertalot }\end{array}$ & $\mathrm{R}$ & $\mathrm{C}$ & $\begin{array}{c}\mathrm{G} 1, \mathrm{G} 2, \mathrm{G} 3, \mathrm{G} 6, \mathrm{N1}, \mathrm{N} 2, \mathrm{N4} 4 \mathrm{N5}, \mathrm{N7}, \mathrm{N8}, \mathrm{N9} \\
\mathrm{N} 10, \mathrm{~N} 11, \mathrm{~N} 15, \mathrm{~N} 16, \mathrm{~N} 17\end{array}$ \\
\hline $\begin{array}{l}\text { Aawicula tripunctata } \\
\text { (Q.EMiiller) Bors. }\end{array}$ & $\mathrm{C}$ & $\mathrm{C}$ & 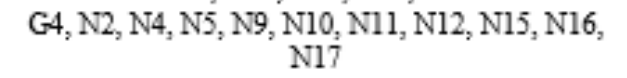 \\
\hline $\begin{array}{l}\text { Alowicula wometa } \\
\text { Kützing }\end{array}$ & $\mathrm{C}$ & $\mathrm{C}$ & $\begin{array}{c}\mathrm{Gl}, \mathrm{G} 2, \mathrm{G} 3, \mathrm{G} 6, \mathrm{N1}, \mathrm{N} 2, \mathrm{~N} 3, \mathrm{~N} 4, \mathrm{~N} 6, \mathrm{~N} 8, \mathrm{~N} 9 \\
\mathrm{N10}, \mathrm{N} 11, \mathrm{~N} 12, \mathrm{~N} 15, \mathrm{~N} 16\end{array}$ \\
\hline $\begin{array}{l}\text { Witsschia archibaldii } \\
\text { Lange-Bertalot }\end{array}$ & $\mathrm{R}$ & $\mathrm{C}$ & $\mathrm{Gl}, \mathrm{G} 2, \mathrm{G} 3, \mathrm{G} 4, \mathrm{~N} 2, \mathrm{~N} 3, \mathrm{~N} 5, \mathrm{~N} 7, \mathrm{~N} 12, \mathrm{~N} 16$ \\
\hline $\begin{array}{l}\text { Nitaschia capitellata } \\
\text { Hustedt }\end{array}$ & $\mathrm{C}$ & $\mathrm{C}$ & $\begin{array}{c}\mathrm{G} 6, \mathrm{N1}, \mathrm{N} 2, \mathrm{~N} 3, \mathrm{N4}, \mathrm{N} 6, \mathrm{N8}, \mathrm{Ng}, \mathrm{N10}, \mathrm{N11}, \mathrm{N12}, \\
\mathrm{N} 13, \mathrm{N14}\end{array}$ \\
\hline $\begin{array}{l}\text { Nitzschia dissipata } \\
\text { (Kützing) Rabenhorst }\end{array}$ & $\mathrm{C}$ & $\mathrm{C}$ & $\begin{array}{c}\mathrm{Gl}, \mathrm{G} 2, \mathrm{G} 3, \mathrm{~N} 1, \mathrm{~N} 2, \mathrm{~N} 4, \mathrm{~N} 5, \mathrm{~N} 6, \mathrm{~N} 7, \mathrm{~N} 9, \mathrm{~N} 10 \\
\mathrm{~N} 11, \mathrm{~N} 12, \mathrm{~N} 15, \mathrm{~N} 16, \mathrm{~N} 17\end{array}$ \\
\hline $\begin{array}{l}\text { Nitaschia fowticala } \\
\text { (Grumowv) Grumowy }\end{array}$ & $\mathrm{C}$ & $\mathrm{C}$ & 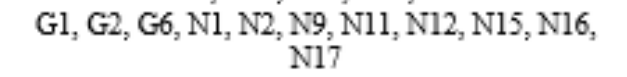 \\
\hline $\begin{array}{l}\text { Nitasshia inconspicua } \\
\text { Sranowi }\end{array}$ & $\mathrm{C}$ & $\mathrm{C}$ & $\begin{array}{c}\mathrm{G} 1, \mathrm{G} 2, \mathrm{G} 3, \mathrm{G} 4, \mathrm{G} 6, \mathrm{N1}, \mathrm{N} 2, \mathrm{~N} 4, \mathrm{~N} 6, \mathrm{~N} 8, \mathrm{~N} 9 \\
\mathrm{~N} 10, \mathrm{~N} 11, \mathrm{~N} 12, \mathrm{~N} 15, \mathrm{~N} 16\end{array}$ \\
\hline $\begin{array}{l}\text { Nitzschia interonedia } \\
\text { Hantzsch }\end{array}$ & $\mathrm{C}$ & $\mathrm{C}$ & $\mathrm{G} 3, \mathrm{G} 6, \mathrm{~N} 1, \mathrm{~N} 2, \mathrm{~N} 3, \mathrm{~N} 4, \mathrm{~N} 8, \mathrm{~N} 9, \mathrm{N11}, \mathrm{N12}, \mathrm{N14}$ \\
\hline $\begin{array}{l}\text { Witzschia linearis } \\
\text { WLSmith }\end{array}$ & $\mathrm{C}$ & $\mathrm{C}$ & $\begin{array}{c}\mathrm{G} 3, \mathrm{G} 6, \mathrm{N1}, \mathrm{N2}, \mathrm{N6}, \mathrm{N7}, \mathrm{N} 8, \mathrm{N9}, \mathrm{N10}, \mathrm{N11}, \mathrm{N15}, \\
\mathrm{N16}\end{array}$ \\
\hline $\begin{array}{l}\text { Nitzschia media } \\
\text { Hantzsch }\end{array}$ & $\mathrm{C}$ & $\mathrm{C}$ & $\mathrm{Gl}, \mathrm{G} 2, \mathrm{G} 3, \mathrm{G} 6, \mathrm{Nl}, \mathrm{N} 2, \mathrm{~N} 4, \mathrm{~N} 5, \mathrm{~N} 15, \mathrm{N16}, \mathrm{N17}$ \\
\hline $\begin{array}{l}\text { Nitaschia pales } \\
\text { (Kützing) WLSmith }\end{array}$ & $\mathrm{C}$ & $\mathrm{C}$ & $\begin{array}{c}\mathrm{G} 1, \mathrm{G} 2, \mathrm{G} 3, \mathrm{G} 6, \mathrm{N1}, \mathrm{N} 2, \mathrm{~N} 3, \mathrm{~N} 4, \mathrm{~N} 6, \mathrm{~N} 7, \mathrm{~N} 8, \mathrm{~N} 9, \\
\mathrm{N10}, \mathrm{N11}, \mathrm{N} 12, \mathrm{~N} 13, \mathrm{~N} 14, \mathrm{~N} 16\end{array}$ \\
\hline $\begin{array}{l}\text { Twoblionolla apiculata } \\
\text { WLGregory }\end{array}$ & $\mathrm{C}$ & $\mathrm{C}$ & $\begin{array}{c}\mathrm{G} 3, \mathrm{~N} 1, \mathrm{~N} 2, \mathrm{~N} 3, \mathrm{~N} 4, \mathrm{~N} 6, \mathrm{~N} 7, \mathrm{~N} 9, \mathrm{~N} 10, \mathrm{~N} 11, \mathrm{~N} 14, \\
\mathrm{~N} 16, \mathrm{~N} 17\end{array}$ \\
\hline
\end{tabular}

Note. C: common ; R: rare 


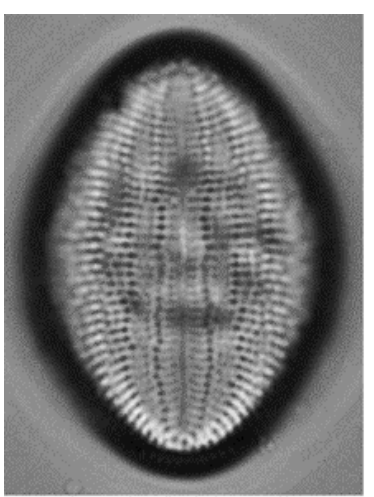

2
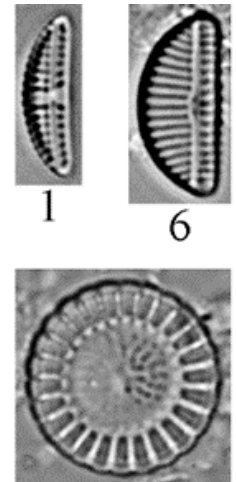

3
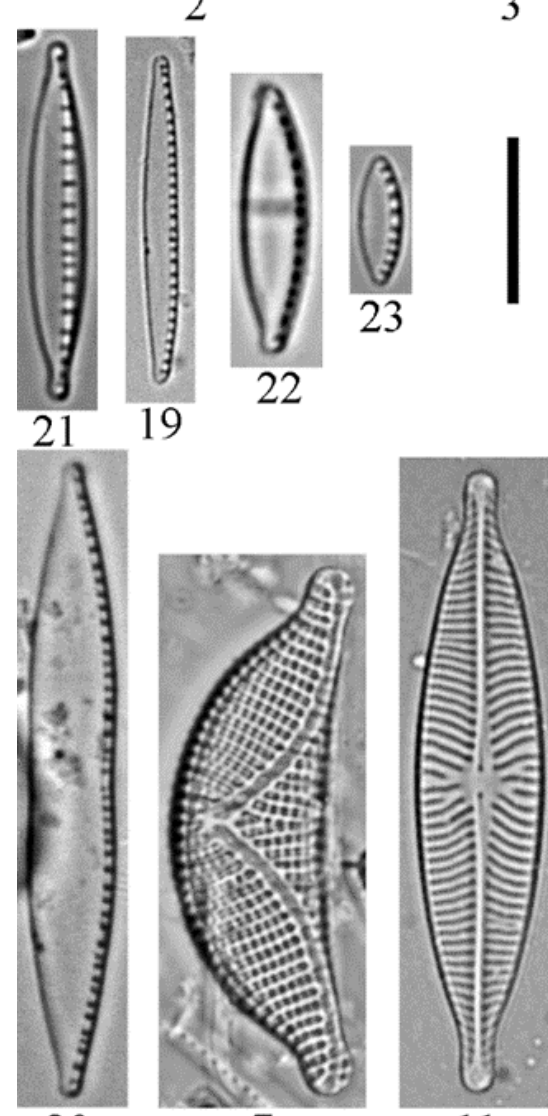

20

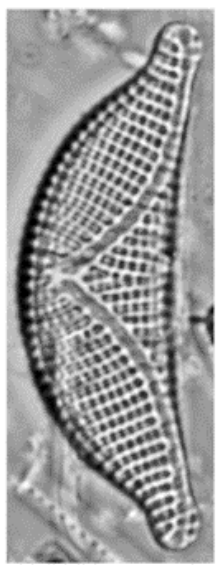

7

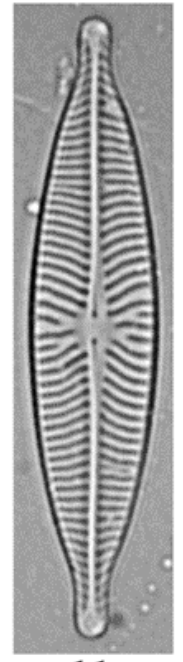

11

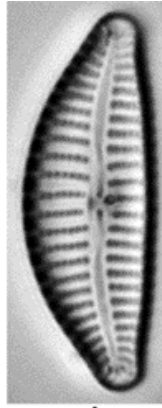

4

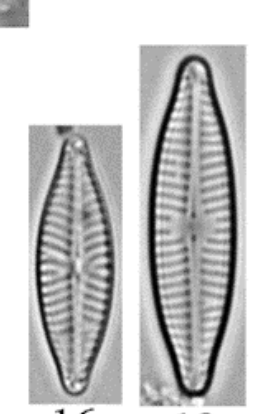

18

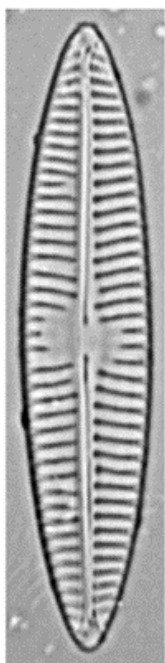

17
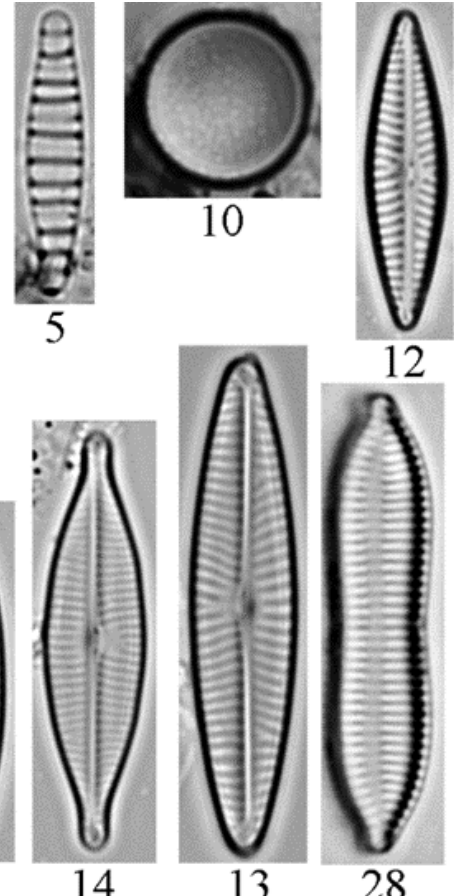

13
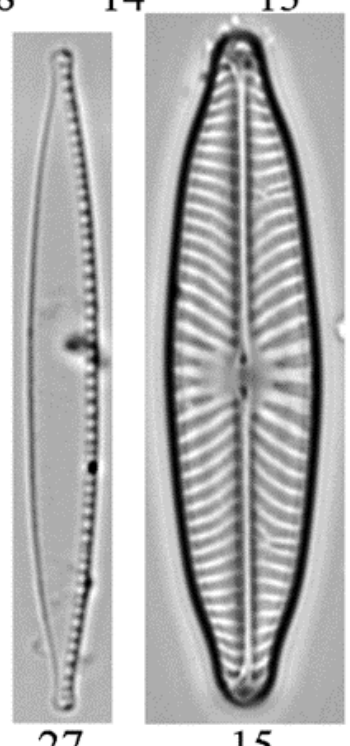

15

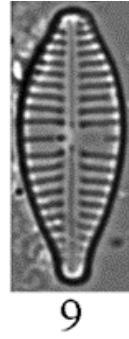

12
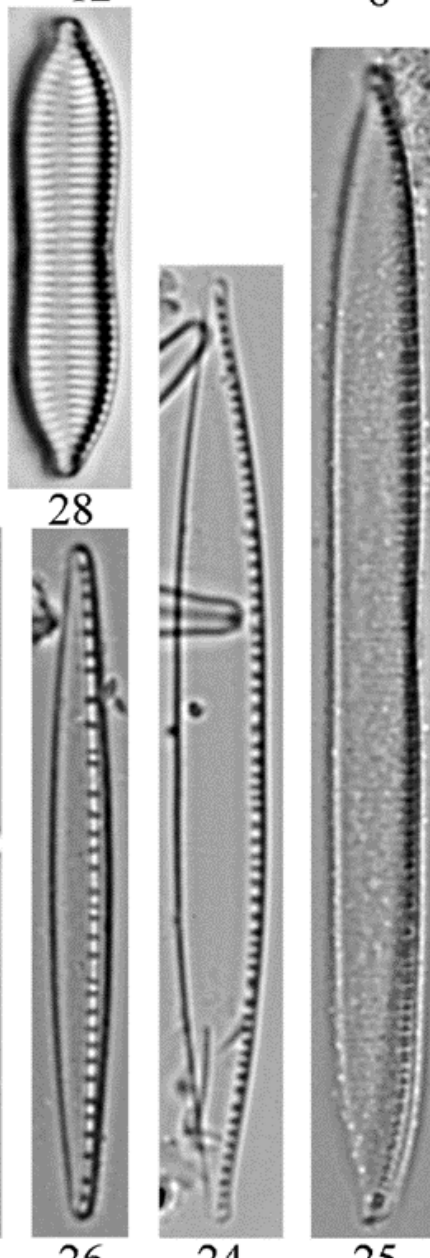

Figure 2. Common Diatoms of Gediz River Basin 1- Amphora pediculus; 2- Cocconeis pediculus; 3- Cyclotella meneghiniana; 4- Cymbella excisa; 5- Diatoma moniliformis; 6- Encyonema minutum; 7- Epithemia sorex; 8- Gomphonema olivaceum; 9- G. parvulum; 10- Melosira varians; 11- Navicula capitatoradiata; 12- $N$. cryptotenella; 13- $N$. erifuga; 14- $N$. gregaria; 15- $N$. novaesiberica; 16- $N$. reichardtiana; 17- $N$. tripunctata; 18- $N$. veneta; 19- Nitzschia archibaldii; 20- N. capitellata; 21- $N$. dissipata; 22- $N$. fonticola; 23- $N$. inconspicua; 24- $N$. intermedia; 25- $N$. linearis; 26- N. media; 27- N. palea; 28- Tryblionella apiculata. Scale bar: $10 \mu \mathrm{m}$. 


\section{Discussion and Conclusion}

Regarding to distribution of the taxa, many of them were also common diatoms in Turkish inland waters (e.g. Amphora ovalis, C. placentula, C. placentula var. euglypta, Craticula accomoda, Cyclostephanos dubius, Cymbella neocistula etc.) (Solak and Wojtal, 2012, Solak et al. 2018b). However, Navicula erifuga, N. reichardtiana, and Nitzschia archibaldii were rarely found in the Turkish waters. Taxonomically, Navicula erifuga close to N.simulata Manguin. However, the latter is radiate striae. The taxon was found in Uluabat Lake (Karacaoğlu et al. 2004; Dalkıran et al. 2017) and Küçük Menderes river basin (Solak et al. 2018a). N. reichardtiana similar to $N$. moskalii Metzeltin et al. and $N$. caterva Hohn \& Hellerman. $N$. moskalii is wider outline while, $N$. caterva has a higher stria density. The taxon was found in Asartepe reservoir (Atıc1 et al. 2010), Küçük Menderes river basin (Solak et al. 2018a) and Kütahya flowing waters (Solak et al. 2016). Nitzschia archibaldii similar to $N$. pumila Hustedt but $N$. pumila is characterised by long protracted ends (Lange -Bertalot et al., 2017). The taxon was found in Küçük Menderes river basin (Solak et al. 2018a). Cocconeis pediculus is close to C. placentula sensu lato. However, C. pediculus has typical striation and structure on RLV. Cymbella excisa was described by Lange-Bertalot (2002). The taxa is very common in the inland waters (identified as $C$. affinis according to Krammer \& Lange-Bertalot (1986). Generally, Nitzschia dissipata is often found together with $N$. media. The latter is longer while, the former has typical axial area.

Ecologically, $A$. pediculus exists in oligo- and $\beta$-mesosaprobic habitats. However, its ecology needs to be revised because the taxon is not easy to identify under LM. $C$. pediculus are characteristics of alkaline, lentic and lotic systems with medium to high trophic levels. C. excisa is cosmopolitan and abundant in the mountains. D. moniliformis tolerates brackish conditions. E. minutum is in anthropogenically little affected habitats. $E$. sorex exists in medium to high trophic levels. G. olivaceum is in mostly eutrophic and moderately electrolyte-rich and, $G$. parvulum is in oligosaprobic and mesosaprobic habitats. Navicula capitatoradiata is in eutrophic to polytrophic running waters and lakes with in weakly brackish waters. $N$. cryptotenella is indicator of $\beta$-mesosaprobic and better conditions. $N$. erifuga is in brackish waters. $N$. gregaria is tolerates up to the $\alpha$ mesosaprobic level. N. veneta is dominant in industrial waste water. Nitzschia amphibia is tolerant to the $\alpha$-mesosaprobic zone while, $N$. capitellata is tolerant to polysaprobic level (Lange-Bertalot et al., 2017; Krammer, 2002).

\section{Acknowledgement}

This study was supported by the Ministry of Agriculture and Forestry, General Directorate of Water Management. We would like to thank to the Directors and the staff of TÜBİTAK MAM Environment and Cleaner Production Institute, who executed Project on Preparation of Gediz River Basin Management Plan. 


\section{References}

Anonymous (2000). Directive 2000/60/EC of the European Parliament and the council of 23 October 2000 establishing a framework for community action in the field of water policy. Official Journal of the European Communities, L327, 1-72.

Atıc1, T., \& Obalı, O. (2010). The diatoms of Asartepe Dam Lake (Ankara), with environmental and some physicochemical properties. Turk J Bot 34: 541-548.

Bey, M. Y. \& Ector, L. (2013). Atlas des diatomées des cours d'eau de la région Rhône-Alpes. Tome 1. Vincennes, France: Agence Française pour la Biodiversité (in French).

Cavalcante, K. P., Tremarin, P. I. \& Ludwig, T. A. V. (2013). Taxonomic studies of centric diatoms (Diatomeae): unusual nanoplanktonic forms and new records for Brazil. Acta Bot Bras 27: 237 - 251.

Çelekli, A., Toudjani, A.A., Lekesiz, H.Ö., Kayhan, \& S., Çetin, T. (2018). Bioassessment of ecological status of three Aegean reservoirs based on phytoplankton metrics. Turkish Journal of Water Science and Management, 1(2), 76-99.

Dalkıran, N., Zünbülgil, B., Karacaoğlu, D \& Dere, Ş. (2016). Uluabat gölü epifitik diyatomelerinin uzun dönemdeki değişimleri. LimnoFish 2(3): 153-163.

Demir, N., Çetin, T., Gök, C., \& Şanal, M. (2017). First biological monitoring in the Akarçay basin according to the Water Framework Directive: phytoplankton and phytobenthos. Turkish Journal of Water Science and Management, 1, 90-107.

Güner, H. (1969). Karagöl’ün makro ve mikro vejetasyonu hakkında ön çalısmalar. - E. Ü. Fen Fakültesi İlmi Raporlar Serisi No. 66. İzmir.

Cirik, S. (1983). Marmara Gölü'ndeki (Manisa) Alg Gruplarının Mevsimsel Belirişleri Üzerinde Bir Araştırma. E.Ü. Fen Fakültesi Dergisi B(I), 574-587.

Cirik, S. (1994). Marmara Gölü Fitoplanktonu IV. Bacillariophyceae. - XII. Ulusal Biyoloji Kongre Kitabl, 315-322. Edirne.

Gezerler-Şipal, U., Balık, S., \& Ustaoğlu, M.R. (1999). Demirköprü Baraj Gölünün (Salihli-Manisa) Fitoplanktonu. II. International Symposium on Aquatic Products, 199-207. İstanbul-Turkey.

Gönülol, A. (2018). Turkish algae electronic publication. Retrieved from http://turkiyealgleri.omu.edu.tr

Håkansson H. (2002). A compilation and evaluation of species in the general Stephanodiscus, Cyclostephanos and Cyclotella with a new genus in the family Stephanodiscaceae. Diatom Res. 17 (1): 1 - 139.

Hofmann G., Werum M. \& Lange-Bertalot H. (2011). Diatomeen im Sußwasser-Benthos von Mitteleuropa. A.R.G. Gantner Verlag K.G., Ruggell.

Hustedt F. (1930). Bacillariophyceae. In: A. Pascher (ed.), Die Susswasser-Flora Mitteleuropas, pp. 1 - 669. Fischer, Jena.

Houk, V., Klee, R., \& Tanaka, H. (2010). Atlas of freshwater centric diatoms with a brief key and descriptions. Part III. Stephanodiscaceae A: Cyclotella, Tertiarius, Discostella. Fottea 10: 1 - 498.

Karacaoğlu, D., Dere Ş., \& Dalkıran N. (2004). A taxonomic study on the phytoplankton of Lake Ulubat (Bursa). Turk J Bot 28: 473-485. 
Kiss, K. T., Klee, R., Ector, L. \& Ács, É. (2012). Centric diatoms of large rivers and tributaries in Hungary. Morphology and biogeographic distribution. Acta Bot Croat 71: 311 - 363.

Krammer, K. (2002). Diatoms of Europe, Diatoms of the European Inland waters and comparable habitats. Cymbella. In: H. Lange-Bertalot (ed.).A.R.G. Gantner Verlag K.G., 584.

Krammer, K. (1997). Die cymbelloiden Diatomeen. Eine Monographie der weltweit bekannten Taxa. Teil 1. Allgemeines und Encyonema part. Bibliotheca Diatomologica Vol. 36. 382 pp.

Krammer, K., \& Lange-Bertalot, H. (1986). Bacillariophyceae. 1. Teil: Naviculaceae In: Ettl, H., J. Gerloff, H. Heynig and D. Mollenhauer (eds.). Süßwasserflora von Mitteleuropa, 2 (1), 1 - 876.

Krammer, K. \& Lange-Bertalot, H. (1991). Bacillariophyceae, 2. Teil: Bacillariaceae, Epithemiaceae, Surirellaceae. Süßwasserfora von Mitteleuropa 2 (2), 1 - 596.

Krammer, K., \& Lange-Bertalot, H. (1991). Bacillariophyceae 3.Teil: Centrales, Fragilariaceae, Eunotiaceae. Süßwasserflora von Mitteleuropa, 2(3), 1 - 576.

Lange-Bertalot, H., Hofmann, G., Werum, M., \& Cantonati, M. (2017). Freshwater Benthic Diatoms of Central Europe: Over 800 Common Species Used in Ecological Assessment.. Koeltz Botanical Books, 942.

Levkov Z. (2009). A mphora sensu lato. In: H. Lange-Bertalot (ed.), Diatoms of Europe 5: 1 - 916.

Patrıck R. \& Reimer C. W. (1966). The Diatoms of the United States, exclusive of Alaska and Hawaii. 1. Fragilariaceae, Eunotiaceae, Achnanthaceae, Naviculaceae. Acad. Nat. Sci. Philadelphia Monogr. 13 (1): $1-688$.

Patrick R. \& Reimer C. W. (1975). The Diatoms of the United States, States, Exclusive of Alaska and Hawaii. 2 (1). Entomoneidaceae, Cymbellaceae, Gomphonemaceae, Epithemaceae. Acad. Nat. Sci. Philadelphia Monogr. 13 (2): 1 - 213.

Solak, C.N., Wojtal, A.Z. (2012). The diatoms in springs and streams of the Türkmen Mountain (Sakarya River Basin), commonly reported from Turkish inland waters. Polish Botanical Journal 57(2), 375-425.

Solak C.N., Kulikovskiy M.S., Kaleli, A., \& Gönülol, A. (2016). Rare and new records of diatoms in Turkey from Kütahya flowing waters, Oceanological and Hydrobiological Studies 45(4): 564-587.

Solak, C.N., Çetin, T., \& Kaleli, A. (2018a). Distribution of benthic diatom (phytobenthos) composition in Küçük Menderes river basin. Turkish Journal of Water Science and Management, 1(2), 2-35.

Solak, C.N., Kulikovskiy, M., Kiss, T.K., Kaleli, A., Kociolek, J.P., \& Ács, É. (2018b). The distribution of centric diatoms in different river catchments in the Anatolian Peninsula, Turkey. Turkish Journal of Botany 42 (1), 100-122.

Swift, E. (1967). Cleaning diatom frustules with ultraviolet radiation and peroxide. Phycologia 6: 161-163.

Tarım ve Orman Bakanlığı, Su Yönetimi Genel Müdürlüğü. (2018). Gediz Nehir Havza Yönetim Planı Hazırlanması Projesi Nihai Raporu. Ankara.

Wojtal, A. Z. (2009). The diatoms of Kobylanka stream near Kraków (Wyżyna Krakowsko-Częstochowska, S Poland). Polish Botanical Journal 54: 129 - 330.

Wojtal, A. Z. \& Kwandrans, J. (2006). Diatoms of the Wyżyna Krakowsko Częstochowska Upland (S Poland) - Coscinodiscophyceae (Thalassiosirophycidae). Polish Botanical Journal 51: 177 - 207.

Yurterin, N., \& Öztürk, M. (2001). Gürle Deresi ve yakın çevresi fitoplanktonları üzerine bir çalışma. $I V$. Ulusal Ekoloji ve Çevre Kongresi Kitabı. Bodrum. 


\section{Extended Turkish Abstract (Genişletilmiş Türkçe Özet)}

\section{Gediz Nehir Havzasındaki Fitobentozun Yaygın Diyatomeleri}

Avrupa Birliği Su Çerçeve Direktifi; su kaynaklarının korunması, geliştirilmesi ve kalitedeki kötüye gidişin engellenmesi amacıyla yürürlüğe konmuştur. Direktifin ana hedefi su kütlelerinde ekolojik iyi duruma ulaşılabilmesidir. Direktifin uygulanabilmesi için her bir havza için nehir havzası yönetim planlarının oluşturulması gerekmektedir. Türkiye'de bulunan 25 nehir havzasından 11 havza (Konya, Susurluk, Meriç-Ergene, Büyük Menderes, Gediz, Yeşilırmak, Akarçay, Batı Akdeniz, Burdur, Küçük Menderes ve Kuzey Ege) için yönetim planı hazırlanması çalışmaları devam etmektedir. Nehir havzası yönetim planlarının hazırlanması sürecinde havzada bulunan su kütlelerinin ekolojik durumunun ortaya konulması gerekmektedir. Ekolojik durumun ortaya konulması için fitoplankton, makrofit, fitobentoz, bentik makroomurgasızlar ve balık gibi biyolojik kalite bileşenleri nehir havzalarında izlenmektedir.

Gediz Havzası coğrafi bakımdan $38^{\circ} 04^{\prime}-39^{\circ}-13^{\prime}$ kuzey enlemleri ile $26^{\circ} 42^{\prime}-29^{\circ} 45^{\prime}$ doğu boylamları arasında yer almaktadır. Havzanın temel su kaynağı olan Gediz Nehrine birçok yan dere katıldıktan sonra Manisa ve Menemen Ovalarını sulayarak denize dökülmektedir. 275 km uzunluğunda olan Gediz Nehri Batı Anadolu'da $17.220 \mathrm{~km}^{2}$ 'lik bir yağış alanından beslenmektedir. Gediz Havzası'nda doğal göl sayısı yok denecek kadar azdır. Havzada yer alan en önemli doğal göl, Akhisar'ın Marmara beldesi yakınlarındaki Marmara Gölü’dür. Marmara Gölünün minimum ve maksimum kotu $73.2-79.2 \mathrm{~m}$, hacmi 8,5-320 hm ${ }^{3}$ tür. Havzada Marmara Gölü’nün dışında doğal göl olarak Gölcük Gölü vardır. Bir krater gölü olan Gölcük Gölü, 80 ha genişliğinde ve $10 \mathrm{~m}$ derinliğindedir. Havzada 5 baraj bulunmaktadır. Havzadaki en büyük baraj 1.022 milyon $\mathrm{m}^{3}$ depolama kapasitesiyle Demirköprü Barajı'dır. Barajın üzerine enerji üretmek üzere HES kurulmuştur. Küçükler Barajları içmesuyu temini için, diğer barajlar sulama, taşkın koruma ve enerji üretimi amaçlı kullanılmaktadır. Demirköprü, Afşar ve Buldan Barajları Manisa'da, Küçükler Barajı ise Uşak’ta yer almaktadir.

AB Su Çerçeve Direktifi kapsamında diyatomeler, fitobentoz biyolojik kalite bileşeninin nehir ve göllerde temsil eden en önemli organizma gruplarındandır. Nehir havzası yönetim planlarının hazırlanması sürecinde yerüstü su kütlelerinde ekolojik durumun ortaya konulması maksadıyla diyatomeler birçok nehir havzasında izlenmektedir. Diyatome örnekleri havzada bulunan 17 nehir (Demirci, Gürdük, Gediz, Gürlevik, Bahçeler, Diken, Derbent, Selendi, Alaşehir, Nif, Sarıkız, Canburt, Murat, Ağıl ve Gürdük akarsuları), 2 göl (Gölcük ve Marmara) ve 4 barajdan (Demirköprü, Küçükler, Buldan, Afşar) Kasım 2017 ve Nisan 2018'de epiliton ve epipelon substratlardan toplanmıştır. Epilitik örnekler nehir yatağında yer alan taşların firçalanmasıyla, epipelik örnekler ise pipet yardımıyla sedimentten toplanmıştır. Organik maddenin frustullerden uzaklaştırılması maksadıyla örnekler $\mathrm{H}_{2} \mathrm{O}_{2}$ ve $\mathrm{HCl}$ ile kaynatılmıştır. Daimi preparatlar Naphrax solüsyonu ile hazırlanmıştır. Diyatomeler mikroskop yardımıyla incelenmiş ve taksonomik literatüre göre teşhis edilmiştir. Havzada yaygın olarak bulunan taksonların görsel katalogları hazırlanmış ve Türkiye florasındaki durumu ile karşılaştırılmıştır. Tür, literatürde \%10 dan fazla yer alıyorsa "yaygın", $\% 10$ dan daha azında yer alıyorsa " $a z$ rastlanır" olarak sınıflandırılmıştır.

Sonuç olarak, Gediz Nehir Havzasında 28 diyatome taksonu yaygın olarak bulunmuştur. Çalışmada tespit edilen taksonların pek çoğu Türkiye nehir ve göllerinde yaygın olarak bulunan taksonlardır. Amphora pediculus (Kützing) Grunow, Nitzschia dissipata (Kützing) Rabenhorst ve Nitzschia inconspicua Grunow örnekleme noktalarında en yaygın tespit edilen türler olmuştur. Ancak, Navicula erifuga Lange-Bertalot, Navicula reichardtiana Lange-Bertalot ve Nitzschia archibaldii Lange-Bertalot Türkiye nehir ve gölleri için nadir bulunan türlerdendir. Navicula erifuga ülkemizde Marmara ve Küçük Menderes nehir havzalarında rastlanmıştır. Diğer bir baskın takson N. reichardtiana ise Sakarya ve Küçük Menderes nehir havlalarında tespit edilmiştir. Türlerin taksonomik özellikleri dikkate alındığında, Cocconeis pediculus ile $C$. placentula birbirine oldukça benzer durumda olup, raphe içermeyen kabuk yapısı (stria yapısı) farklılık göstermektedir. Cymbella excisa ise yine yaygın diyatomelerden biridir. Bu tür ilk olarak C. affinis olarak tanımlanmıştır. Türlerin ekolojik özellikleri gözönüne alındığında, A mphora pediculus temiz sularda bulunur. Cymbella excisa az kirli alkalinsularda bulunur. Diatoma moniliformis hafif tuzlu suların karakteristik türlerinden birisidir. 
Gomphonema olivaceumdaha ziyade ötrofik ve orta derecede iletkenlik seviyesine sahip sularda bulunur. Diğer yaygın bir tür olan G. parvulum ise ekolojik toleransı geniş türlerden biridir. Navicula capitatoradiata daha ziyade kirli sularda yayılış gösterir. Navicula gregaria ve Nitzschia amphibia kirliliğe nispeten toleranslı türlerden biridir. Nitschia capitellata ise çok kirli sulara toleranslı türler arasındadır. 